\title{
FROBENIUS ALGEBRAS AND SKEIN MODULES OF SURFACES IN 3-MANIFOLDS
}

\author{
UWE KAISER \\ Department of Mathematics, Boise State University \\ 1910 University Drive, Boise, ID 83725-1555 U.S.A. \\ E-mail: kaiser@math.boisestate.edu
}

\begin{abstract}
For each (commutative) Frobenius algebra there is defined a skein module of surfaces embedded in a given 3-manifold and bounding a prescribed curve system in the boundary. The skein relations are local and generate the kernel of a certain natural extension of the corresponding topological quantum field theory. In particular the skein module of the 3-ball is isomorphic to the ground ring of the Frobenius algebra. We prove a presentation theorem for the skein module with generators incompressible surfaces colored by elements of a generating set of the Frobenius algebra, and with relations determined by tubing geometry in the manifold and relations of the algebra.
\end{abstract}

1. Introduction. In $[\mathrm{P}]$ the idea of algebraic topology based on knots originated. The idea is to study quotients of formal linear combinations of links by local relations that allow computation of the modules and understanding of the interplay between link theory and geometric topology of the 3-manifold. Most interesting skein modules of links in 3-manifolds are based on skein relations with the property that the modules of the 3-ball are isomorphic to the ground ring. Typically one considers relations suggested by link polynomials like the Jones, Homflypt or Kauffman bracket polynomials. Understanding the structure of these modules for general 3-manifolds is an important question which is related to basic problems of quantum topology like the volume conjecture [L]. From their definition as quantum invariants all these polynomial link invariants have operator-valued extensions as 1-dimensional embedded topological quantum field theories [T1], [T2].

It is well-known that theories of surfaces in 3-manifolds provide powerful tools towards the understanding of 3-manifold topology. So it seems surprising that general skein the-

2000 Mathematics Subject Classification: Primary 57M25; Secondary 57M35, 57R42.

Key words and phrases: 3-manifold, incompressible surface, Frobenius algebra, skein module, Bar-Natan relation, topological quantum field theory.

The paper is in final form and no version of it will be published elsewhere. 
ories of surfaces in 3-manifolds have not yet been developed. Probably the reason is that embeddings of surfaces appear to be too rigid. In fact, the local theory is not interesting because there are just no nontrivial local embeddings of surfaces. Contrary, knot theory should be interesting for 3-manifolds because of the interplay of local knotting and linking with the global topology and geometry of 3-manifolds.

Recently the study of skein relations between surfaces was suggested by Bar-Natan [B]. It has been put forward into the framework of general 3-manifolds by Asaeda and Frohman $[\mathrm{AF}]$. The structure of skein modules and related categories have been studied by Gad Naot [N1], [N2]. In [Kh] Khovanov initiates the discussion of skein relations between surfaces originating from more general Frobenius algebras. The abstract 2-dimensional topological quantum field theories defined from Frobenius algebras turned out to be very interesting for Khovanov theory. Their role in general 3-manifold theory (and hopefully the relation to 4-manifold theories) remains to be explored, see $[\mathrm{AF}],[\mathrm{FK}]$. But we will see that globalizing skein relations of surfaces yields structures which, actually not very surprisingly, measure global embedding phenomena of surfaces in 3-manifolds in a nice nontrivial way.

Until now, because of the justified emphasis on the Khovanov theory background, many results have been proven separately for different skein relations. It is the goal of this paper to initiate a discussion of a large class of skein modules defined from embedded surfaces in 3-manifolds. We will see that many properties of modules defined using Frobenius algebras are closely related to the corresponding 2-dimensional topological quantum field theories. In fact we will show that the skein relations naturally emerge from a discussion of the kernel of a suitably extended topological quantum field theory. Also many results proven for specific skein relations will hold in broad generality. In section 9 we prove a presentation theorem which shows that in the case of free Frobenius algebra the module can be generated by surfaces colored with elements of the basis, and relations defined from tubing geometry of the 3-manifold. For example the tunnel number of a knot naturally appears in the discussion of skein modules of surfaces in knot complements. In general the relations of our skein modules of surfaces in 3-manifolds with respect to natural generating sets, can be expressed through the difficult geometric problem of understanding the different ways to compress a given surface in a 3-manifold.

Frobenius algebras also naturally appear in the study of quantum cohomology, and through the concept of Frobenius manifolds are related with loop spaces, symplectic geometry and Landau-Ginzburg models. See $[\mathrm{H}]$ for a good introduction.

Most of the ideas that we discuss here are already present in the work of Asaeda and Frohman $[\mathrm{AF}]$. But we will elaborate on various subtleties emerging in the discussion of skein modules of surfaces in 3-manifolds from general Frobenius algebras, which do not seem to appear elsewhere in the published literature. The author is aware that some of the material in this paper is folklore in the community of Khovanov theory researchers.

2. Frobenius algebras and Frobenius systems. For further details about some of the following algebraic concepts see $[\mathrm{K}]$ and $[\mathrm{Kh}]$. We would like to point out that what we call a Frobenius algebra is called a Frobenius system in [Kh]. 
Throughout let $R$ be a commutative ring with 1 . All tensor products are over $R$, so $\otimes=\otimes_{R}$.

A Frobenius algebra $\mathcal{F}=(R, A, \Delta, \varepsilon)$ is a commutative ring $A$ with 1 and inclusion $\iota: R \rightarrow A$ such that $\iota(1)=1$. Thus $A$ is an $R$-algebra with product

$$
\mu: A \otimes A \rightarrow A
$$

and unit $\iota$. Also

$$
\Delta: A \rightarrow A \otimes A
$$

is a cocommutative and coassociative coproduct, which is an $A$-bimodule map, and has as counit the $R$-module map $\varepsilon: A \rightarrow R$.

Note that the counit condition means

$$
(\varepsilon \otimes I d) \circ \Delta=I d,
$$

where we identify $R \otimes A=A \otimes R=A$ using the restrictions of $\mu$.

The $R$-algebra $A$ is a special case of a Frobenius extension of $R$, see $[\mathrm{K}]$. Note that all our Frobenius algebras are commutative by definition.

If we write $\Delta(1)=\sum_{i=1}^{r} u_{i} \otimes v_{i}$ for $u_{i}, v_{i} \in A$ then (1) and cocommutativity imply that for all $a \in A$ :

$$
a=\sum_{i=1}^{r} \varepsilon\left(a u_{i}\right) v_{i}=\sum_{i=1}^{r} \varepsilon\left(a v_{i}\right) u_{i}
$$

In particular, $A$ is a finitely generated $R$-module with the two generating sets $\left\{u_{i} \mid i=\right.$ $1, \ldots r\}$ and $\left\{v_{i} \mid i=1, \ldots, r\right\}$.

Conversely, each set $\left\{\left(u_{i}, v_{i}\right) \mid i=1, \ldots r\right\} \subset A \times A$ such that $\sum_{i=1}^{r} u_{i} \otimes v_{i}=\sum_{i=1}^{r} v_{i} \otimes$ $u_{i}$ and (2) holds for a given $\varepsilon$, defines a cocommutative and coassociative $A$-bimodule coproduct by

$$
\Delta(1)=\sum_{i=1}^{r} u_{i} \otimes v_{i}
$$

with counit $\varepsilon$.

Following $[\mathrm{K}]$ we call $\left(R, A,\left\{\left(u_{i}, v_{i}\right) \mid i=1, \ldots, r\right\}, \varepsilon\right)$ a Frobenius system. Each Frobenius system determines a Frobenius algebra, and conversely for each Frobenius algebra there exists a not necessarily unique Frobenius system. The rank of a Frobenius system is defined by $\sum_{i=1}^{r} \varepsilon\left(u_{i} v_{i}\right)=\varepsilon \mu \Delta(1) \in R$. This only depends on the underlying Frobenius algebra.

Remark 2.1. (a) Let $\left\{\left(u_{i}, v_{i}\right) \mid i=1, \ldots r\right\}$ define a Frobenius system on the $R$-algebra $A$ and let $\left\{w_{i} \mid i=1, \ldots, s\right\}$ be a generating set of $A$. Then there exist $\lambda_{i j} \in R$ for $i=1, \ldots, r$ and $j=1, \ldots, s$ such that $v_{i}=\sum_{j=1}^{s} \lambda_{i j} w_{j}$.Thus $\left\{\left(z_{j}, w_{j}\right) \mid j=1, \ldots s\right\}$ with $z_{j}=\sum_{i=1}^{r} \lambda_{i j} u_{i}$ define a (usually different) Frobenius system with the same underlying Frobenius algebra.

(b) Often one has the additional assumption that $A$ is a projective $R$-module, i.e. summand of a free $R$-module. This condition plays a role in discussions about the structure theory of topological quantum field theories associated to Frobenius algebras. It will not at all be relevant for our discussions. 
(c) To avoid redundancies one can assume in the definition of Frobenius systems that for each subset $J \subset\{1, \ldots, r\}$

$$
\sum_{i \in J} u_{i} \otimes v_{i} \neq 0
$$

The natural $R$-module map

$$
A \ni a \mapsto(A \ni b \mapsto \varepsilon(b a))
$$

defines an $R$-isomorphism

$$
A \rightarrow A^{*}=\operatorname{Hom}_{R}(A, R) .
$$

If $A$ is a free $R$-module then we can find a Frobenius system for it with both $\left\{u_{i}\right\}$ and $\left\{v_{i}\right\} R$-bases. Then by (3), applied to the functionals mapping some $u_{i}$ to 1 and all other $u_{j}$ to 0 , it follows that there exists a dual basis $\left\{w_{i}\right\}$ of $A$ such that

$$
\varepsilon\left(u_{i} w_{j}\right)=\delta_{i j},
$$

where $\delta_{i j}=1$ for $i=j$ and $=0$ for $i \neq j$. A free Frobenius algebra is a Frobenius algebra with $A$ a free $R$-module. For $a \in A$ let $\mu_{a}: A \rightarrow A$ be defined by

$$
\mu_{a}(c)=\mu(a \otimes c)=a c
$$

for all $c \in A$.

In Khovanov theory gradings are important. A Frobenius algebra is graded if $A$ is a graded ring (a graded abelian group with $\mu$ a degree 0 mapping) such that all structure maps are graded.

EXAmple 2.2. (a) Following $[\mathrm{K}]$ we define the universal rank two Frobenius structure with $R=\mathbb{Z}[h, t], A=R[X] /\left(X^{2}-h X-t\right)$ and $\varepsilon(1)=0, \varepsilon(X)=1$, and $\{(1, X-h),(X, 1)\}$ defining the system (corresponding to the bases $\{1, X\}$ and $\{X-h, 1\}$ ). Then

$$
\Delta(1)=1 \otimes X+X \otimes 1-h 1 \otimes 1 .
$$

It follows from $A$-bilinearity that:

$$
\begin{aligned}
\Delta(X)=\Delta(1) X & =(1 \otimes X+X \otimes 1-h(1 \otimes 1)) X \\
& =1 \otimes X^{2}+X \otimes X-h(1 \otimes X) \\
& =1 \otimes(h X+t)+X \otimes X-h(1 \otimes X) \\
& =X \otimes X+t(1 \otimes 1) .
\end{aligned}
$$

Note that the values $\varepsilon_{n}:=\varepsilon\left(X^{n}\right) \in R$ are recursively determined by $\varepsilon_{0}=0, \varepsilon_{1}=1$ and

$$
\varepsilon_{n+2}=h \varepsilon_{n+1}+t \varepsilon_{n}
$$

for all $n \geq 0$. The corresponding universal Frobenius algebra $\mathcal{F}_{\mathbf{U}}$ is graded by $\operatorname{deg}(X)=1$ and $\operatorname{deg}(h)=2, \operatorname{deg}(t)=4$.

(b) Let $R=\mathbb{Z}$ and $G$ a finite abelian group. Then the group algebra $\mathbb{Z}[G]$ is a Frobenius extension of $\mathbb{Z}$. The natural Frobenius algebra $\mathcal{F}_{G}$ is defined by $\Delta(1)=\sum_{g} g^{-1} \otimes g$, with $\varepsilon(g)=0$ for $g \neq e$ and $\varepsilon(e)=1$, where $e \in G$ is the identity of the group. This is a free Frobenius algebra with basis $G$. 
(c) Given a Frobenius algebra $\mathcal{F}=(R, A, \Delta, \varepsilon)$ and some invertible element $y \in A$ there is defined the twisted Frobenius algebra $\mathcal{F}^{y}:=\left(R, A, \Delta^{\prime}, \varepsilon^{\prime}\right)$ by $\Delta^{\prime}=\Delta \circ \mu_{y^{-1}}$ and $\varepsilon^{\prime}=\varepsilon \circ \mu_{y}$. It is known that any two Frobenius algebras defining the same Frobenius extension differ by twisting, see $[\mathrm{K}]$, Theorem 1.6.

Given a Frobenius algebra $\mathcal{F}$ we let $M(\mathcal{F})$ denote the symmetric monoidal subcategory of the category of all (projective) $R$-modules with objects $A^{\otimes r}, r \geq 0\left(A^{0}:=R\right)$, which is generated by tensor product and composition from the structure morphisms $\mu, \iota, \Delta, \varepsilon, \tau$ and $\mu_{a}$ for all $a \in A$. Note that all structure morphisms are $R$-module maps, and only $\iota$ and $\varepsilon$ are not $A$-module respectively $A$-bimodule maps. The set of objects is naturally identified with the set of natural numbers $\{0,1,2, \ldots\}$.

3. Extending 2-dimensional topological quantum field theories. It is well-known that Frobenius algebras (with ground ring $R$ ) define (orientable) 2-dimensional topological quantum field theories with values in the category of $R$-modules. Let $C o b$ denote the category with objects 1 -dimensional closed manifolds, standardly embedded in $\mathbb{R}^{2}$ (thus the objects are naturally identified with the natural numbers). The morphisms are isotopy classes of orientable 2-manifolds, embedded in $\mathbb{R}^{N} \times[0,1]$, for $N$ large, bounding corresponding input respectively output 1 -manifolds in $\mathbb{R}^{2} \times 1$ respectively $\mathbb{R}^{2} \times 0$. The monoidal structure is defined by disjoint union and the symmetric structure is defined by the switch surface defined from two cylinders.

For $r \geq 0$ the topological quantum field theory assigns to the 1-manifold $\amalg_{r} S^{1}$ ( $\amalg$ is disjoint union) the $R$-module $A^{\otimes r}$ with $A^{\otimes 0}:=R$. The structure maps $\Delta, \mu, \varepsilon, \iota$ and $\tau$ of the Frobenius algebra are assigned to the pair of pants as you wear it, the pair of pants turned upside down, the cap (local maximum), the cap upside down (local minimum), and the disjoint union of two cylinders switching the order of two input and output circles.

This defines the symmetric monoidal functor:

$$
F(\mathcal{F}): C o b \rightarrow M(\mathcal{F})
$$

with values in the symmetric monoidal subcategory of $M(\mathcal{F})$, which is monoidally generated by the structure maps of $\mathcal{F}$ (in fact into the subcategory of $\mathcal{M}(\mathcal{F})$ generated by the structure morphisms except the $\mu_{a}$ but we will need the extension soon).

Recall the usual way to calculate the $R$-module morphism assigned to a surface by $F(\mathcal{F})$ : Approximate the height function $S \subset \mathbb{R}^{N} \times[0,1] \rightarrow[0,1]$ by a Morse function with values in $[0,1]$. We assume that it takes value 1 on input circles and value 0 on output circles. Then break up $[0,1]$ into subintervals, each containing only one nondegenerate critical point. Then the $R$-module map is determined by the monoidal functor property of the topological quantum field theory from the values on the standard surfaces. Finally represent possible permutations by sequences of transpositions of neighboring circles. Note that this in general will lead to a difficult calculation if carried out explicitly.

It is essentially Bar-Natan's observation [B], at least in a special case, that the calculation can be carried out locally using skein relations. This is important if one tries to understand what properties are necessary such that Khovanov homology can be defined when using a particular Frobenius algebra, see [Kh]. 
We will extend some of his ideas in this framework to the general case of Frobenius algebras.

Definition 3.1. Let $A$ be a commutative ring with 1 . Then we define the category $\operatorname{Cob}(A)$ as follows: The objects are the same as those of $C o b$. The morphisms are isotopy classes of surfaces with components colored by elements of $A$. Using the multiplication of $A$ the composition of morphisms is defined. Moreover $\operatorname{Cob} \subset \operatorname{Cob}(A)$ is the obvious subcategory of those morphisms with all colors 1 .

The morphisms of $\operatorname{Cob}(A)$ can also be considered as pairs $(S, w)$ where $S$ is an object of $C o b$ and $w \in H^{0}(S, A)=\operatorname{Hom}\left(H_{0}(S), A\right)$ is the coloring.

THEOREM 3.2. The functor $\mathcal{F}$ naturally extends to a symmetric monoidal functor, also denoted

$$
F(\mathcal{F}): \operatorname{Cob}(A) \rightarrow M(\mathcal{F}) .
$$

Proof. Using monoidal structures it suffices to define the functor for connected surfaces in $\operatorname{Cob}(A)$. Suppose a connected orientable surface $S$ is colored by $a \in A$. First assume that the boundary of $S$ is not empty. Then choose an input or output circle and preor postcompose by the corresponding map $I d \otimes \ldots \otimes \mu_{a} \otimes \ldots \otimes I d$. We know that $\Delta$ respectively $\mu$ are $A$-bimodule maps and cocommutative respectively commutative. Thus it does not matter which input or output circle is chosen. In fact, if $a=a_{1} a_{2} \ldots a_{r+s}$ is any factorization then the factors can be distributed in an arbitrary way to the $r+s$ boundary circles. If $S$ is a closed nonempty connected surface choose a Morse function on $S$ as above and consider a nonempty preimage of a regular value. Then the corresponding $R$-module map $R \rightarrow R$ assigned to $S$ is computed by breaking the surface and the corresponding morphism $R \rightarrow A^{\otimes r} \rightarrow R$ where $r \geq 1$ is the number of circles at the regular value. The extended topological quantum field theory now inserts $\mu_{a} \otimes I d^{r-1}: A^{\otimes r} \rightarrow A^{\otimes r}$ between the two $R$-module maps. It is not hard to show that the above construction is well-defined. A multiplication morphism can be moved through a connected surface using the fact that $\mu$ and $\Delta$ are $A$-bimodule maps. For example consider the pair of pants morphism $A \rightarrow A \otimes A$. If the pant is colored $a$ we associate to it the morphism

$$
\Delta \mu_{a}=\left(\mu_{a} \otimes I d\right) \Delta=\left(I d \otimes \mu_{a}\right) \Delta .
$$

Note that these identities follow from $\Delta(b a)=\Delta(b) a=\Delta(a b)=a \Delta(b)$ for all $b \in A$, and express the commutativity of $\mu$ and the $A$-bimodule property of $\Delta$.

Let $\operatorname{Cob}_{R}(A)$ denote the category where the morphism sets of $\operatorname{Cob}(A)$ are replaced by the free $R$-modules with bases the sets of morphisms of $\operatorname{Cob}(A)$. Then the above functor extends by $R$-linearity uniquely to the functor:

$$
F(\mathcal{F}): \operatorname{Cob}_{R}(A) \rightarrow M(\mathcal{F}) .
$$

The definitions in the following section are motivated from analyzing the kernel of this functor $F(\mathcal{F})$, see section 5 .

4. Skein modules of surfaces in 3-manifolds. Let $M$ be a 3-manifold and let $\alpha \subset$ $\partial M$ be a closed 1-manifold. Let $\amalg$ denote disjoint union and $|X|$ denote the number of components of a topological space $X$. 
Let $\mathcal{F}=(R, A, \Delta, \varepsilon)$ be a Frobenius algebra as in section 2. Choose a Frobenius system for $\mathcal{F}$ with $\Delta(1)=\sum_{i=1}^{r} u_{i} \otimes v_{i}$ and $u_{i}, v_{i} \in A$.

For each subset $\mathfrak{a} \subset A$ let $\mathcal{S}(M, \alpha, \mathfrak{a})$ denote the set of isotopy classes of properly embedded surfaces $S$ in $M$ with boundary $\alpha$ (isotopy relative to the boundary), and components colored by elements of $\mathfrak{a}$. The elements of $\mathcal{S}(M, \alpha, \mathfrak{a})$ are called $\mathfrak{a}$-colored surfaces in $M$ bounding $\alpha$. For $\alpha=\emptyset$ we also consider the empty surface $\emptyset$ (with empty color) as an element of $\mathcal{S}(M, \emptyset, \mathfrak{a})$.

The most important cases are $\mathcal{S}(M, \alpha):=\mathcal{S}(M, \alpha, A)$, often just denoted $\mathcal{S}$, and $S(M, \alpha, 1)$. The second set will always be identified with the usual set of isotopy classes of surfaces in $M$ bounding $\alpha$. Formally, elements of $\mathcal{S}(M, \alpha)$ are pairs $(S, w)$, where $w \in H^{0}(S ; A)$ is a locally constant continous map $w: S \rightarrow A$.

For each multplicatively closed $\mathfrak{a} \subset A$ we can also consider several colors on a component. Then the commutative product of $A$ associates a unique element of $\mathcal{S}(M, \alpha, \mathfrak{a})$. There is the map defined by forgetting colors:

$$
\mathfrak{f}: S(M, \alpha, A) \rightarrow S(M, \alpha, 1),
$$

and $\mathfrak{f}^{-1}(S)$ is in one-to-one correspondence with $A^{|S|}$ for $S \in \mathcal{S}(M, \alpha, 1)$, at least up to possible reordering of components by isotopy.

In order to describe relations we use the following local patch notation. We let $(a)$ represent a local patch of a surface colored by $a \in A$. This can be the color of a whole component, or there can be other colors on the same component, depending on the situation. Also in $(a)\left(a^{\prime}\right)$ the two patches may be on the same or distinct components. Sometimes we will also allow additional colors outside of the surface patch. Thus the component of the patch $(a)$ can be colored by a product of $a$ with other elements of $A$.

Consider the $R$-submodule $\mathfrak{R}:=\mathfrak{R}(M, \alpha ; \mathcal{F})$ of $R \mathcal{S}$ that is generated by the following three types of elements.

(1) (R-multi-linearity) For all $a_{1}, a_{2} \in A$ we have

$$
\left(a_{1}+a_{2}\right)-\left(a_{1}\right)-\left(a_{2}\right) \in \mathfrak{R},
$$

and for $a \in A$ and $r \in R$ we have

$$
(r a)-r(a)
$$

(Notice the similarity with the definition of tensor products).

(2) (sphere relations) Let $S$ be a surface in $\mathcal{S}$ where the component colored by $a$ is a 2 -sphere bounding a 3-ball in $M$. Then the difference (in $R \mathcal{S}$ ) between $S$ and the product with $\varepsilon(a)$ of the surface we get by omitting the colored sphere component from (a), is also in $\Re$.

(3) (neck cuttings) Let $\gamma$ be a simple closed curve in the interior of a surface $S$ such that $\gamma$ bounds a disk $D$ in $M$ with $D \cap F=\gamma$. (Such a curve is automatically two-sided. This remark is due to Charlie Frohman.) Then

$$
(a)-\sum_{i=1}^{r}\left(a u_{i}\right)\left(v_{i}\right) \in \mathfrak{R} .
$$

Here the surface on the right hand side results by replacing the annular neighborhood of $\gamma$ by two embedded disks in $M$, which are the indicated pieces. 
If $\gamma$ is separating then the two disks are on different components, and we say this is a separating neck cutting. If $\gamma$ is non-separating then

$$
(a)-(\mu \Delta(a)) \in \Re,
$$

where the right hand surface is defined by cutting the handle, and we have a nonseparating neck cutting.

We want to prove that the submodule $\mathfrak{R}$ defined as above only depends on the Frobenius algebra and not on the Frobenius system giving the elements $\left\{\left(u_{i}, v_{i}\right)\right\}$ used in (3). In order to see this consider colored surfaces in $\mathcal{S}$ with two disjointly embedded ordered disks on the surface. Let $\widetilde{\mathcal{S}}$ denote the resulting set of isotopy classes. There is defined a mapping

$$
(A \otimes A) \times \widetilde{\mathcal{S}} \rightarrow C^{\prime},
$$

where $C^{\prime}$ is the quotient of $R \mathcal{S}$ by the $R$-linearity relations, as follows. Given $\sum_{j=1}^{s} x_{i} \otimes$ $y_{i} \in A \otimes A$ and $S$ with the distinguished disks colored $a$ and 1, define the image by

$$
\sum_{i=1}^{s}\left(a_{1} x_{i}\right)\left(a_{2} y_{i}\right) .
$$

where we use the local notation as before. Note that this is well-defined in $C^{\prime}$ and only depends on the element $\sum_{i=1}^{s} x_{i} \otimes y_{i} \in A \otimes A$. Now a curve $\gamma$ on a surface like in the neck-cutting relation defines several liftings to $\widetilde{\mathcal{S}}$, differing by the order of disks and the different ways of splitting $a=a_{1} a_{2}$ if $a$ is the coloring of the component containing $\gamma$. But because of the cocommutativity of $\Delta$ and the $A$-bimodule property the resulting element of $\mathcal{C}^{\prime}$ does not depend on the choice of lifting, and the elements in $C^{\prime}$ determine $\Re$ up to relations (1) and (2).

Thus $\mathfrak{R}$ is determined by the Frobenius system, and we have the following:

Definition 4.1. For each Frobenius algebra $\mathcal{F}$ and 3 -manifold $M$ with closed 1-manfold $\alpha \subset \partial M$ there is defined the skein module

$$
C(M, \alpha ; \mathcal{F}):=R \mathcal{S}(M, \alpha, A) / \mathfrak{R}(M, \alpha ; \mathcal{F}) .
$$

Usually we will omit the coefficient system from the notation. Let $C(M)=C(M ; \mathcal{F})$ if $\alpha=\emptyset$.

The image of $(S, w)$ in $C(M, \alpha)$ is usually also denoted $(S, w)$, and we write $S$ for $(S, 1)$, a surface with all components colored by $1 \in A$. We just write 1 instead of $\emptyset$ in linear combinations.

REMARK 4.2. (a) Obviously $\mathcal{S}(M, \alpha, \mathfrak{a}) \neq \emptyset$ for $\mathfrak{a} \neq \emptyset$ if and only if $\mathcal{S}(M, \alpha, 1) \neq \emptyset$. If $\mathcal{S}(M, \alpha, A)=\emptyset$ then $C(M, \alpha)=0$. But it is not a priori clear whether $\mathcal{S}(M, \alpha, 1) \neq \emptyset$ implies $C(M, \alpha) \neq 0$. We will discuss some of these issues in section 7 .

(b) The reason to allow nonorientable surface in the definition of skein modules is motivated from Khovanov theory on surfaces [M], [TT]. It is easy to define orientable or oriented versions of the skein modules because the relations (1)-(3) above preserve orientations. So there are obvious definitions of those skein module, for each Frobenius algebra. For example let $\tilde{C}(M, \tilde{\alpha})$ be the corresponding quotient of the free $R$-module generated 
by oriented properly embedded surfaces in $M$ bounding the oriented 1-manifold $\tilde{\alpha} \subset \partial M$ by the oriented version of the relations (1)-(3).

(c) Given $S \in S(M, \alpha, 1)$ then the quotient of $R \mathfrak{f}^{-1}(S)$ by the submodule spanned by relations (1) is a certain quotient of $A^{\otimes|S|}$, the quotient coming from some action of the symmetric group corresponding to possible reordering of colored components by isotopy. The structure of skein modules of surfaces in 3-manifolds as above very much is determined by this algebraic relation.

(d) Let $\mathcal{F}$ be graded such that $\varepsilon$ has degree -2 and $\Delta$ has degree 2 . Then the skein module $C(M, \alpha)$ is graded using $\operatorname{deg}(S, w)=-\chi(S)+\sum_{j} \operatorname{deg}\left(a_{j}\right)$, where the sum is over all components of the colored surface $S$ with $w$ assigning $a_{j}$ to the $j$-th component.

(e) Let $y \in R$ be invertible. Then multiplication by $y$ defines an isomorphism of modules

$$
C(M, \alpha ; \mathcal{F}) \rightarrow C\left(M, \alpha, \mathcal{F}^{y}\right) .
$$

But if $y \in A \backslash R$ then the situation is more difficult because in general the skein modules are not $A$-modules in a natural way. Note that for all $y \in A$ there is defined the natural bijective map on the level of isotopy of surfaces:

$$
\mathcal{S}(M, \alpha) \rightarrow \mathcal{S}(M, \alpha)
$$

by $(S, w) \mapsto(S, y w)$, i.e. by changing the colors of all components. This is obviously not compatible with the geometric relations.

Let $C_{1}(M, \alpha) \subset C(M, \alpha)$ be the submodule generated by the image of $S(M, \alpha, 1)$ in $C(M, \alpha)$.

Proposition 4.3. If the powers of $\mu(\Delta(1)) \in A$ form a set of $R$-generators of the algebra A then $C_{1}(M, \alpha)=C(M, \alpha)$.

Proof. This follows because deleting a handle from a component of a surface in $M$ using a neck cutting amounts to multiplication of the color of that component by $\mu(\Delta(1))$. Thus we can eliminate the colors of components by adding trivial handles.

If 4.3 applies then we say that the skein modules are geometric. But often 4.3 does not apply, even in the Bar-Natan case with $R=\mathbb{Z}$ because $\mu(\Delta(1))=2 X$. The powers of this element oviously do not generate $A$. If we replace $R$ by $\mathbb{Z}\left[\frac{1}{2}\right]$, and change $A$ correspondingly, then the skein modules become geometric. For the universal rank two Frobenius algebra $\mu(\Delta(1))=2 X-h$. Again, if we replace $R=\mathbb{Z}[h, t]$ by $\mathbb{Z}\left[\frac{1}{2}, h, t\right]$ then the module becomes geometric. But then, using some easy isomorphisms, one can show that the Frobenius algebra will just be the Gad Naot system (with an additional parameter $h$ added), see [Kh], Examples 1.

We will discuss the relation of our skein modules above with the examples that have been previously discussed in the literature in section 8 .

The following is immediate from the definitions.

Proposition 4.4. Suppose that the 3-manifold $M$ has $k$ components $M_{i}, i=1, \ldots, k$ and $(M, \alpha)=\amalg_{1 \leq i \leq k}\left(M_{i}, \alpha_{i}\right), \alpha_{i} \subset \partial M_{i}$ for $i=1, \ldots, k$. Then

$$
C(M, \alpha)=\bigotimes_{i=1}^{k} \mathcal{C}\left(M_{i}, \alpha_{i}\right)
$$




\section{2-dimensional topological quantum field theory and skein modules. Con-} sider the skein modules

$$
\mathcal{C}(r, s):=C\left(D^{2} \times I, r u \times\{1\} \amalg s u \times\{0\}\right),
$$

where for integers $r \geq 0$ let $r u$ denote the union of $r$ standardly embedded trivial circles in the disk $D^{2}$.

There are $R$-module maps from $\mathcal{S}\left(D^{2} \times I, r u \times 1 \amalg s u \times 0, A\right)$ onto the sets of morphisms of the category $\operatorname{Cob}_{R}(A)$. The relations in the definition of skein modules can be applied abstractly to the morphism sets of $\operatorname{Cob}_{R}(A)$ to define a quotient category $\operatorname{Cob}(\mathcal{F})$. The objects of this category are still the objects of $C o b$ but the morphism sets are the quotients of the morphism modules of $C_{o b}(A)$ by $R$-linearity and abstract sphere- and neck cutting relations. The resulting quotient category is denoted $\operatorname{Cob}(\mathcal{F})$.

There are induced $R$-module maps

$$
\mathcal{C}(r, s) \rightarrow \operatorname{Cob}(r, s)
$$

where $\operatorname{Cob}(r, s)$ is the morphism module from $r$ to $s$ circles in the category $\operatorname{Cob}(\mathcal{F})$. We want to prove that these are in fact isomorphisms.

THEOREM 5.1. The functor $F(\mathcal{F})$ defined in Theorem 3.2, more precisely its extension to $\operatorname{Cob}_{R}(A)$, factors through $\operatorname{Cob}(\mathcal{F})$ and defines an isomorphism of categories

$$
\operatorname{Cob}(\mathcal{F}) \rightarrow M(\mathcal{F})
$$

Proof. The observation that the functor factors through the quotient category $\operatorname{Cob}(\mathcal{F})$ is by construction. For example, a sphere colored $a \in A$ will map to the $R$-morphism $R \rightarrow R$ given by $\varepsilon \circ \mu_{a} \circ \iota=\varepsilon(a) I d_{R}$. But this is also the image of $\varepsilon(a) \emptyset$, where $\emptyset$ is the empty surface (which maps to the identity $I d_{R}: R \rightarrow R$ ). A similar argument using equation (2) from section 2 proves that the neck cutting relations are in the kernel of the topological quantum field theory. Thus

$$
F(\mathcal{F}): \operatorname{Cob}(\mathcal{F}) \rightarrow M(\mathcal{F})
$$

is well-defined and onto by the very definition of $M(\mathcal{F})$. We have to prove injectivity of the sets of morphisms. For $r, s \geq 0$ consider the $R$-morphism

$$
\phi_{r, s}: A^{\otimes(r+s)} \rightarrow \operatorname{Cob}(r, s)
$$

defined by mapping $a_{1} \otimes a_{r} \otimes b_{1} \otimes b_{s}$ to the surface consisting of $r+s$ disks colored with $a_{1}, \ldots, b_{s}$. This is well-defined in $\operatorname{Cob}(r, s)$ because of the $R$-multi-linearity relations. Consider the composition with $F(\mathcal{F})$. This is the $R$-morphism:

$$
\begin{gathered}
A^{\otimes(r+s)} \rightarrow \operatorname{Hom}\left(A^{r}, A^{s}\right) \\
a_{1} \otimes \ldots \otimes a_{r} \otimes b_{1} \otimes \ldots \otimes b_{s} \mapsto\left(a_{1}^{\prime} \otimes \ldots \otimes a_{r}^{\prime} \mapsto \varepsilon\left(a_{1} a_{1}^{\prime}\right) \ldots \varepsilon\left(a_{r} a_{r}^{\prime}\right) b_{1} \otimes \ldots \otimes b_{s}\right) .
\end{gathered}
$$

It is not hard to see that these $R$-morphisms are injective. To keep notation simple we show the case $r=2, s=1$. Let $\sum_{k=1}^{N} a_{k} \otimes b_{k} \otimes c_{k}$ be in the kernel of the composition. It is mapped to the $R$-morphism $A \otimes A \rightarrow A$ defined by

$$
a \otimes b \mapsto \sum_{k=1}^{N} \varepsilon\left(a a_{k}\right) \varepsilon\left(b b_{k}\right) c_{k},
$$


which we assume to be the trivial map. Now for $k=1, \ldots, N$ we can write using equation (2) of section 2:

$$
a_{k}=\sum_{i_{k}=1}^{r} \varepsilon\left(a_{k} u_{i_{k}}\right) v_{i_{k}} \quad \text { and } \quad b_{k}=\sum_{j_{k}=1}^{r} \varepsilon\left(b_{k} u_{j_{k}}\right) v_{j_{k}} .
$$

Thus

$$
\sum_{k=1}^{N} a_{k} \otimes b_{k} \otimes c_{k}=\sum_{k=1}^{N} \sum_{i_{k}=1}^{r} \sum_{j_{k}=1}^{r} \varepsilon\left(a_{k} u_{i_{k}}\right) \varepsilon\left(b_{k} u_{j_{k}}\right) v_{i_{k}} \otimes v_{j_{k}} \otimes c_{k} .
$$

But substituting $a=u_{i_{k}}$ and $b=u_{j_{k}}$ for fixed values of $i_{k}, j_{k} \in\{1, \ldots, r\}$ we get

$$
\sum_{k=1}^{N} \varepsilon\left(a_{k} u_{i_{k}}\right) \varepsilon\left(b_{k} u_{j_{k}}\right) c_{k}=0
$$

and thus the sum is vanishing.

It follows from the proof of 5.1 that the $R$-module maps $\phi_{r, s}$ are isomorphisms. This proves the second half of

Corollary 5.2. For all $r, s$ we have natural isomorphisms

$$
\mathcal{C}(r, s) \cong \operatorname{Cob}(r, s) \cong A^{\otimes(r+s)} .
$$

Under these isomorphisms the composition morphisms

$$
\operatorname{Cob}(s, t) \otimes \operatorname{Cob}(r, s) \rightarrow \operatorname{Cob}(r, t)
$$

correspond to

$$
\left(b_{1}^{\prime} \otimes b_{s}^{\prime} \otimes c_{1} \otimes c_{t}\right) \otimes\left(a_{1} \otimes a_{r} \otimes b_{1} \otimes b_{s}\right) \mapsto \varepsilon\left(b_{1} b_{1}^{\prime}\right) \ldots \varepsilon\left(b_{s} b_{s}^{\prime}\right)\left(a_{1} \otimes a_{r} \otimes c_{1} \otimes c_{t}\right) .
$$

Proof. Since all surfaces embedded in $D^{2} \times I$ are completely compressible we can apply neck cutting and sphere relations to see that the modules $\mathcal{C}(r, s)$ also are also $R$-generated by $A$-colored disks bounding the corresponding input and output circles. So the $R$-module maps

$$
\psi_{r, s}: A^{\otimes(r+s)} \rightarrow \mathcal{C}(r, s)
$$

defined by coloring disks as above is onto, and factors the $R$-module map $\phi_{r, s}$ from above. Because $\phi_{r, s}$ is injective, $\psi_{r, s}$ is also injective. Thus the projections $c(r, s) \rightarrow \operatorname{Cob}(r, s)$ are isomorphisms.

Theorem 5.1. and its corollaries contain several results concerning Bar-Natan modules previously discussed in the literature, see e.g. [N1], [N2]. For example the cases $s=0$ respectively $r=s=0$ give the following results:

COROllary 5.3. The skein module $C\left(D^{3}, r u ; \mathcal{F}\right)$ is isomorphic to $A^{\otimes r}$ for all Frobenius algebras $\mathcal{F}$. In particular for $r=0$ we have

$$
C\left(D^{3}\right) \cong R .
$$

The image of a connected surface of genus $g$ colored by $a \in A$ is

$$
\varepsilon\left(\left(\mu(\Delta(1))^{g} a\right) \in R\right.
$$

and the isomorphism maps disjoint unions to products. 
6. Some properties of skein modules. The following result has been proved for Bar-Natan modules in [FK]. For $i=1,2$ let $M_{i}$ be two 3 -manifolds with disks $D_{i} \subset \partial M_{i}$ and closed 1-manifolds $\alpha_{i} \subset \partial M_{i} \backslash D_{i}$. Then we can define the boundary connected sum $M_{1} \sharp M_{2}$ with the closed 1-manifold $\alpha:=\alpha_{1} \cup \alpha_{2}$ in its boundary. Note that $M_{i} \subset M_{1} \sharp M_{2}$ for $i=1,2$.

THEOREM 6.1.

$$
C\left(M_{1} \sharp M_{2}, \alpha_{1} \cup \alpha_{2}\right) \cong C\left(M_{1}, \alpha_{1}\right) \otimes C\left(M_{2}, \alpha_{2}\right) .
$$

Proof. Let $M:=M_{1} \sharp M_{2}$. Let $D \subset M_{1} \sharp M_{2}$ be the disk along which the connected sum has been formed. First we define an $R$-module map:

$$
\phi: C\left(M_{1}, \alpha_{1}\right) \otimes C\left(M_{2}, \alpha_{2}\right) \rightarrow C(M, \alpha) .
$$

Since $C\left(M_{1}, \alpha_{1}\right) \otimes C\left(M_{2}, \alpha_{2}\right)$ is $R$-generated by the image of $S\left(M_{1}, \alpha_{1}, A\right) \otimes S\left(M_{2}, \alpha_{2}, A\right)$ under the tensor product of the natural projections, we can let $\phi$ map the image of $S_{1} \otimes S_{2}$ to the disjoint union $S_{1} \amalg S_{2} \subset M_{1} \sharp M_{2}$, which bounds $\alpha$. It is immediate from the definitions that this map is well-defined. The map is surjective because we can apply neck cutting relations to all the circles in the transversal intersection $D \cap S$ for a surface $S \in \mathcal{S}(M, \alpha)$. Note that the set of intersection circles is partially ordered by nesting. The neck cuttings have to be performed starting from innermost circles. In order to show that $\phi$ is injective we will construct the inverse $R$-module map:

$$
\psi: C(M, \alpha) \rightarrow C\left(M_{1}, \alpha_{1}\right) \otimes C\left(M_{2}, \alpha_{2}\right)
$$

as follows. For each generating (colored) surface $S \subset M$ bounding $\alpha$ apply neck cuttings to the transverse intersection $S \cap D$. We have to prove that the resulting linear combination in $C\left(M_{1}, \alpha_{1}\right) \otimes C\left(M_{2}, \alpha_{2}\right)$ does not depend on the choice of surface in its isotopy class relative boundary. Now isotopies $S_{t}$ where the intersection $D \cap S_{t}$ is only changes by isotopy in $D$ will give rise to isotopies of the cut surfaces in $M_{1}$ respectively $M_{2}$. Otherwise it suffices to consider elementary isotopies with a single time parameter $t_{0}$ for which the surface $S_{t_{0}}$ is not transversal as follows (or its inverse): (i) this surface touches the disk at some point, and thus pushing through the disk will create a new intersection circle, (ii) the surface intersects $D$ in a saddle, so for $t<t_{0}$ the intersection consists of two circles that are connected to a single circle when pushing the saddle through $D$ from $M_{1}$ into $M_{2}$. Because the cutting is done in order of the partial order of circles we can assume that all these circles are innermost. Now in case (i) there is a 2 -sphere bounding a 3 -ball in $M$ and application of the neck cutting does not contribute. In the second case we compare the result of the two neck cuttings for $t<t_{0}$ with the neck cutting along the one circle for $t>t_{0}$. But an additonal neck cutting in $M_{2}$ gives the same result. What we have to check can, because of $R$-multi-inearity, be expressed in the patch notation by:

$$
\sum_{i, j}\left(a u_{i} u_{j}\right)\left(v_{j}\right)\left(v_{i}\right)=\sum_{i j}\left(a u_{i}\right)\left(u_{j}\right)\left(v_{i} v_{j}\right)
$$

for all $a \in A$. But this is the relation

$$
(I d \otimes \Delta) \circ \Delta=(\Delta \otimes I d) \circ \Delta,
$$

which follows from cocommutativity of $\Delta$ and $A$-bilinearity. It corresponds to switching 
two consecutive saddle points of a surface in the topological quantum field theory associated to $\mathcal{F}$. Finally compatibility with relations (1)-(3) is easly seen from locality of the relations.(For the neck cuttings one has to use the disks in $M$ bounding the cutting curves on $M$ to find isotopies to push the disks away from $D$.) Thus $\psi$ is well-defined, and $\psi \circ \phi=I d$ proves that $\phi$ is also injective.

The special case of $M_{2}$ a 3-ball, with a trivial loop $u$ in its boundary disjoint from the connected sum disk, proves by induction on $r$ the following:

Corollary 6.2. Let $r \geq 0$. Suppose $r u$ is any disjoint union of $r$ simple closed loops in $\partial M$, each of which bounds a disk in $\partial M$ and is contained in the complement of a 1-manifold $\alpha \subset \partial M$. Then there is an isomorphism of $R$-modules

$$
C(M, \alpha \amalg r u) \cong C(M, \alpha) \otimes A^{\otimes r} .
$$

Remark 6.3. For $\mathcal{F}$ free one can also give give a direct proof of 6.1 . without reference to 5.3. (which in fact reproves 5.3 in this case). Again it suffices to prove the case $r=1$. Let $\mathfrak{b}=\left\{u_{i} \mid i=1, \ldots, r\right\}$ be a $R$-basis of $A$ and $\left\{w_{j} \mid j=1, \ldots, r\right\}$ be a dual basis such that $\varepsilon\left(u_{i} w_{j}\right)=\delta_{i j}$. Using 8.1. we can identify $C(M, \alpha)$ and $C(M, \alpha, \mathfrak{b})$ (see 8.1.). Then define the $R$-module map

$$
\phi: \mathcal{S}(M, \alpha, \mathfrak{b}) \otimes A \rightarrow \mathcal{S}(M, \alpha \amalg u, \mathfrak{b})
$$

by

$$
\phi((S, w) \otimes b)=\left(S^{\prime}, w^{\prime}\right)
$$

for $b \in \mathfrak{b}$. Here $S^{\prime}$ is the disjoint union of $S$ with a disk $D$ bounding $u$ in a collar neighborhood of $\partial M$ and define $w^{\prime}\left|S=w, w^{\prime}\right| D=b$. It is important to observe that elements of $R$ can move freely between components of $S$ and the disk using the $R$ linearity relations. Because the skein relations are local there is the induced $R$-module map $C(M, \alpha) \otimes A \rightarrow \mathcal{C}(M, \alpha \amalg u)$. This map is onto because we can apply the neck cutting relations to elements of $\mathcal{S}(M, \alpha \amalg u, \mathfrak{b})$ to expand in linear combinations of $\mathfrak{b}$ colored surfaces given by a marked disk bounding $u$ and a marked surface bounding $\alpha$. It suffices to prove that $\phi$ is injective. Let $\phi\left(\sum_{i=1}^{r} x_{i} \otimes u_{i}\right)=0$ with $x_{i} \in C(M, \alpha)$ for and $i=1, \ldots, r$. Then define for $j=1, \ldots, r$ :

$$
\psi_{j}: C(M, \alpha \amalg u) \rightarrow C(M, \alpha)
$$

on marked surfaces $(S, w) \in C(M, \alpha \amalg u)$ by adding a collar to $M$ along $\partial M$, capping off $u$ by a disk colored $w_{j}$ and adding a cylinder over $\omega$. This results in a well-defined element of $C(M, \alpha)$. It follows from the definitions that for $j=1, \ldots, r$

$$
\psi_{j}\left(\phi\left(\sum_{i=1}^{r} x_{i} \otimes u_{i}\right)\right)=\sum_{i=1}^{r} x_{i} \varepsilon\left(u_{i} w_{j}\right)=x_{j}
$$

Thus $\phi$ is also injective.

The proof of the following result is very similar to the proof of 6.2 .

Proposition 6.4. For $(M, \alpha)$ let $\left(M^{\prime}, \alpha\right)$ be the result of attaching a 1-handle $H$ to $M$ along $\partial M$ such that $H \subset \partial M \backslash \alpha$. Then the inclusion $M \subset M^{\prime}$ induces the isomorphism

$$
C(M, \alpha) \rightarrow C\left(M^{\prime}, \alpha\right) \text {. }
$$


Corollary 6.5. $C\left(H_{g}\right) \cong R$ for $H_{g}$ the genus $g$ handlebody.

It follows that $C\left(H_{g}, r u\right) \cong A^{\otimes r}$ for trivial curves in $\partial H_{g}$. Also $C\left(H_{g}, \mu\right) \cong A$ for a meridian $\mu$ follows by attaching a solid 1-handles to $\left(D^{3}, u\right)$ suitably.

In general it seems difficult but interesting to determine $C(\Sigma \times I, \alpha)$ for $\alpha \subset \partial(\Sigma \times I)$, see also 7.5. Note that is relatively easy to describe the beviour of the modules under attaching 1-handles while it is difficult to study the effect of attaching 2-handles. This is in a certain way dual to the situation for skein modules of links in 3-manifolds where attaching 2-handles admits a conceptual description (handle slides induce relations) while the skein modules of handlebodies are hard to describe and represent difficult objects, see $[\mathrm{P}]$.

7. Naturality properties of the skein modules. First we discuss a universal coefficient theorem. Let $\mathcal{F}=(R, A, \Delta, \varepsilon)$ and $\mathcal{F}^{\prime}=\left(R^{\prime}, A^{\prime}, \Delta^{\prime}, \varepsilon^{\prime}\right)$ be two Frobenius system. Let $\phi: \mathcal{F} \rightarrow \mathcal{F}^{\prime}$ be a morphism of Frobenius algebras. This is an $R$-algebra morphism $A \rightarrow A^{\prime}$, also denoted $\phi$, which commutes with all obvious diagrams relating the structure maps of $\mathcal{F}$ with those of $\mathcal{F}^{\prime}$. For example $\iota^{\prime} \circ \phi \mid R=\phi \circ \iota$, and

$$
(\phi \otimes \phi) \circ \Delta=\Delta^{\prime} \circ \phi .
$$

Note that $\phi$ induces on $R^{\prime}$ the structure of $R$-module. A morphism of Frobenius algebras is called epimorphism if both morphisms $A \rightarrow A^{\prime}$ and $R \rightarrow R^{\prime}$ are onto. An epimorphism of Frobenius algebras is called coefficient induced if $A^{\prime}=A \otimes_{R} R^{\prime}$ and all structure maps of $\left(R^{\prime}, A^{\prime}, \Delta^{\prime}, \varepsilon^{\prime}\right)$ are induced via tensor product $\otimes_{R}$ in the obvious way from $(R, A, \Delta, \varepsilon)$.

ExAmple 7.1. Let $G, G^{\prime}$ be two finite groups. Then each group homomorphism $\phi: G \rightarrow$ $G^{\prime}$ extends to an epimorphism of the Frobenius algebras $\mathcal{F}_{G} \rightarrow \mathcal{F}_{G^{\prime}}$ defined by the natural morphism of group algebras. In particular this applies to the morphism $G \rightarrow\{1\}$.

The first claim of the following proposition is obvious, and the second one is proven in the same way as the universal coefficient theorem for link skein modules, see $[\mathrm{P}]$.

Proposition 7.2. Let $\phi: \mathcal{F} \rightarrow \mathcal{F}^{\prime}$ be an epimorphism of Frobenius algebras. Then $\phi$ induces an epimorphism of $R$-modules

$$
C(M, \alpha ; \mathcal{F}) \rightarrow C\left(M, \alpha ; \mathcal{F}^{\prime}\right),
$$

where $C\left(M, \alpha ; \mathcal{F}^{\prime}\right)$ is considered as $R$-module via $R \rightarrow R^{\prime}$. If $\phi$ is coefficient induced then there is a natural isomorphism

$$
C\left(M, \alpha ; \mathcal{F}^{\prime}\right) \cong C(M, \alpha ; \mathcal{F}) \otimes_{R} R^{\prime}
$$

Next consider for $\alpha \subset \partial M$ the exact sequence

$$
H_{2}\left(\alpha ; \mathbb{Z}_{2}\right) \rightarrow H_{2}\left(M ; \mathbb{Z}_{2}\right) \rightarrow H_{2}\left(M, \alpha ; \mathbb{Z}_{2}\right) \stackrel{\partial}{\longrightarrow} H_{1}\left(\alpha ; \mathbb{Z}_{2}\right) \stackrel{j_{*}}{\longrightarrow} H_{1}\left(M ; \mathbb{Z}_{2}\right)
$$

and

$$
\partial^{-1}[\alpha] \subset H_{2}\left(M, \alpha ; \mathbb{Z}_{2}\right)=H_{2}\left(M, N(\alpha) ; \mathbb{Z}_{2}\right),
$$

where $[\alpha]$ is the fundamental class and $N(\alpha)$ is a neighborhood of $\alpha$ in $\partial M$.

By the Pontrjagin-Thom construction the set

$$
\partial^{-1}[\alpha] \subset H_{2}\left(M, N(\alpha) ; \mathbb{Z}_{2}\right) \cong H^{1}\left(M / N(\alpha) ; \mathbb{Z}_{2}\right) \cong\left[M / N(\alpha), \mathbb{R} P^{\infty}\right]
$$


can be interpreted as the set of bordism classes of surfaces in $M$ with boundary $\mathbb{Z}_{2^{-}}$ homologous to $\alpha$. It is possible that $\partial^{-1}[\alpha]=\emptyset$, in which case $S(M, \alpha, A)=\emptyset$ and $C(M, \alpha)=0$. (For example take for $\alpha$ an odd number of longitudes in the boundary of a solid torus.) On the other hand, each element in $\mathcal{S}(M, \alpha, A)$ determines an element in $H_{2}\left(M, N(\alpha) ; \mathbb{Z}_{2}\right)$.

The skein modules of surfaces always decompose with respect to $\mathbb{Z}_{2}$-homology.

Proposition 7.3.

$$
C(M, \alpha) \cong \bigoplus_{a \in \partial^{-1}[\alpha]} C_{a}(M, \alpha),
$$

where $C_{a}(M, \alpha)$ is the skein module spanned by all surfaces representing $a \in H_{2}\left(M, \alpha ; \mathbb{Z}_{2}\right)$. If $j_{*}[\alpha]=0$ then $\partial^{-1}[\alpha]$ is in one-to-one correspondence with $H_{2}\left(M ; \mathbb{Z}_{2}\right)$. Otherwise $\partial^{-1}[\alpha]=\emptyset$.

Proof. This follows from the fact that the skein relations imply $\mathbb{Z}_{2}$-homology of the underlying surfaces and the exactness of the homology sequence.

For skein modules of surfaces bounding oriented curve systems the analogous result holds with $\mathbb{Z}$-homology replacing $\mathbb{Z}_{2}$-homology. The discussion of skein modules of orientable surfaces bounding curve systems leads to the study the image of $\mathbb{Z}$-homology in $\mathbb{Z}_{2}$-homology.

Suppose $\alpha$ is two-sided on $\partial M$ such that $N(\alpha)$ is a union of tori. Then any surface with boundary $\mathbb{Z}_{2}$-homologous to $\alpha$ can be modified by glueing annuli to pairs of parallel boundary components to a surface bounding $\alpha$. For each $a \in \partial^{-1}[\alpha]$ there exists an element of $S(M, \alpha, A)$ with homology class $a$.

Proposition 7.4. Suppose $S_{1}, S_{2} \in S(M, \alpha)$ are two surfaces with

$$
\left[S_{1}\right]=\left[S_{2}\right] \in H_{2}\left(M, \alpha ; \mathbb{Z}_{2}\right) .
$$

Then by adding tubes to $S_{1}$ and $S_{2}$ we get surfaces $S_{1}^{\prime}$ and $S_{2}^{\prime}$ which are isotopic relative boundary.

Proof. It obviously suffices to consider the case $M$ connected. The basic idea is classical and often used in the discussion of $S$-equivalence of Seifert surfaces (see [Ka] for a detailed account) with the main argument given in [KL]. Actually a quite subtle argument is necessary here to prove first that $S_{1}$ and $S_{2}$ bound a 3-manifold $W \subset M \times I$ such that $W \cap(\partial M \times I)=\alpha \times I$. This is shown by obstruction theory of maps into $\mathbb{R} P^{\infty}$. Then by adding 1-handles (respectively tubes), we can make both $S_{1}, S_{2}$ connected. Finally a handle cancellation argument, see $[\mathrm{KL}]$, proves the claim.

Let $d: M \rightarrow M$ be a diffeomorphism of $M$. Then $d$ induces an $R$-module map

$$
d_{*}: C(M, \alpha) \rightarrow C(M, h(\alpha)) .
$$

The induced morphism is an automorphism for diffeomorphisms that are constant on the boundary. In particular the modules $C(M, \alpha)$ are representations of the mapping class group Diff $(M, \partial M)$ of isotopy classes of diffeomorphisms that are the identity on $\partial M$. 
EXAMPLE 7.5. For the understanding of Khovanov theory of diagrams on oriented surfaces $\Sigma$ the calculation of the skein modules

$$
C(\Sigma \times I, \alpha \times\{1\} \cup \beta \times\{0\})
$$

for $\alpha, \beta \subset \Sigma$ two closed 1-manifolds is important, at least for the universal rank 2 Frobenius system. Note that by 6.2 . we can assume that both $\alpha$ and $\beta$ are essential. The skein modules here are also morphism sets of a certain category $\operatorname{Cob}(\Sigma, \mathcal{F})$, essentially first essentially defined by Turner and Turaev in [TT] (and denoted $\mathcal{U} \operatorname{Cob}(\Sigma)$ there to emphasize the possibility of unorientable surfaces). The understanding of the $R$-algebras

$$
C(\Sigma \times I, \alpha \times\{0,1\})
$$

seems interesting for a possible construction of the tautological topological quantum field theories suggested by Bar-Natan in [B]. The first important cases obviously are the two algebras for $\alpha$ a simple closed non-separating curve respectively $\alpha$ empty. See $[\mathrm{FK}]$ for calculations in the Bar-Natan case.

8. Presentations of skein modules and presentations of $A$. First we show how a presentation of the $R$-module $A$ induces a presentation of the $R$-module $C(M, \alpha)$.

Let $\mathfrak{g} \subset A$ be a generating set of $A$ as $R$-module and $\mathfrak{r} \subset R \mathfrak{g}$ be a set of relations such that

$$
A \cong R \mathfrak{g} / \operatorname{span}(\mathfrak{r}),
$$

where $\operatorname{span}(\mathfrak{r})$ is the $R$-submodule of $R \mathfrak{g}$ generated by $\mathfrak{r}$. Let $\mathfrak{p}$ denote the presentation $(\mathfrak{g}, \mathfrak{r})$.

Recall that

$$
\mathcal{S}(M, \alpha, \mathfrak{g}) \subset \mathcal{S}(M, \alpha, A)
$$

is the set of isotopy classes of surfaces in $M$ bounding $\alpha$, with the colors of all components in $\mathfrak{g}$. If a component is colored by several elements of $A$ then we always assume that their product is in $\mathfrak{g}$, even if $\mathfrak{g}$ is not multiplicatively closed. The elements of $S(M, \alpha, \mathfrak{g})$ are called marked surfaces with respect to $\mathfrak{g}$. Then let

$$
\mathfrak{R}(M, \alpha, \mathfrak{p}) \subset R \mathcal{S}(M, \alpha, \mathfrak{g})
$$

to be the submodule generated by (using the patch notation from section 4 ):

$\left(1^{\prime}\right) \sum_{i=1}^{s} r_{i}\left(g_{i}\right)=0$ where $\sum_{i=1}^{s} r_{i} g_{i} \in \mathfrak{r}$ and $g_{i} \in \mathfrak{g}$ and $r_{i} \in R$ for $i=1, \ldots, s$;

$\left(2^{\prime}\right)$ sphere relations for all 2-spheres bounding 3-balls which are colored by elements of $\mathfrak{g}$;

$\left(3^{\prime}\right)$ neck cutting relations for which the component of the neck is colored by some element of $\mathfrak{g}$ and the right hand side is expanded using $R$-linearity into a linear combination of $\mathfrak{g}$-colored surfcaes.

Let $C(M, \alpha ; \mathfrak{p})$ be the quotient of $R \mathcal{S}(M, \alpha, \mathfrak{g})$ by $\mathfrak{R}(M, \alpha, \mathfrak{p})$.

THEOREM 8.1. The inclusion

$$
\mathcal{S}(M, \alpha, \mathfrak{g}) \subset \mathcal{S}(M, \alpha, A)
$$

induces the isomorphism

$$
C(M, \alpha, \mathfrak{p}) \cong C(M, \alpha)
$$


Proof. Obviously $\mathfrak{R}(M, \alpha, \mathfrak{p})$ is contained in the kernel of the induced $R$-module map

$$
R \mathcal{S}(M, \alpha, \mathfrak{g}) \rightarrow C(M, \alpha)
$$

which is onto because $\mathfrak{g}$ is a generating set and the $R$-linearity relations hold in $C(M, \alpha)$. Let $\phi$ be the $R$-module map induced on the quotient module. Construct an inverse of $\phi$ by first defining

$$
\mathcal{S}(M, \alpha, A) \rightarrow C(M, \alpha, \mathfrak{p})
$$

using expansion of elements of $A$ in terms of $\mathfrak{g}$ and $R$-multi-linearity. It is clear from the definitions that $\mathfrak{R}=\mathfrak{R}(M, \alpha ; \mathcal{F}) \subset R \mathcal{S}(M, \alpha, A)$ is contained in the kernel of this mapping. The induced $R$-module map proves that $\phi$ is an isomorphism.

REMARK 8.2. (a) Suppose $\mathfrak{g}$ is closed under multiplication in $A$. Then the neck cutting relations $\left(3^{\prime}\right)$ above are just special neck cutting relations (3) (there is no need to expand the right hand side).

(b) If $\mathfrak{g}$ is a basis and $\mathfrak{r}=0$ then there are no relations $\left(1^{\prime}\right)$.

Example 8.3. (a) Consider the universal rank 2 Frobenius algebra $\mathcal{F}_{\mathbf{U}}$, so $A$ is a free $R$-module with basis $\{1, X\}$. Let $\mathfrak{g}=\left\{X^{i}, i=0,1,2, \ldots\right\}$ be the multiplicatively closed generating set with relation $X^{2}=h X+t$. Then $\mathcal{S}(M, \alpha, \mathfrak{g})$ is the set of dotted surfaces (with possibly many dots) where a dot on a component corresponds to the color $X$. The relations $\left(1^{\prime}\right)-\left(3^{\prime}\right)$ above in this case have been used in $[\mathrm{C}]$ to describe the universal Bar-Natan module. But of course one can give a reduced description based on the basis $\{1, X\}$ and relations $\left(2^{\prime}\right)$ and $\left(3^{\prime}\right)$ alone.

(b) If we reduce the universal rank 2 Frobenius algebra by $t=h=0$ we have $R=\mathbb{Z}$ and the relation that each surface with a component with two dots is 0 because $X^{2}=0$ (two dot relation). This is the Frobenius system $\mathcal{F}_{B N}$ studied in [AF] and first described in [B]. It is relevant to the categorification of the Jones polynomial as originally described by Khovanov. In this case the module $C(M, \alpha)$ is the Bar-Natan module of the 3-manifold $M$ with respect to $\alpha \subset \partial M$. Those modules have been essentially computed for $\alpha=\emptyset$ and $M$ a closed Seifert fibred 3-manifold in [AF].

(c) In [N1], [N2] Gad Naot defines the following version of Bar-Natan modules. He takes the quotient of the free abelian group generated by the set of elements $\mathcal{S}(M, \alpha, A)$ with all components colored 1 (the usual isotopy classes of surfaces) by the Bar-Natan relations: $\left(2^{\prime \prime}\right)$ if a surfaces has a 2 -sphere component then the surface is trivial, $\left(3^{\prime \prime}\right) 2 S=S_{+}+S_{-}$, where $S$ is a surface with a neck given by a compressible loop $\gamma$ on $S, S_{ \pm}$are the two surfaces which result by cutting as before but with adding an additional trivial 1-handle inserted into the left respectively right hand disk. Then he proves that adding two 1handles into a component does not depend on the component. Even though his argument is given in the abstract setting it is easy to see that it can be generalized to our skein modules of embedded surfaces in 3-manifolds using connectedness and the possibility appearances of unorientable surfaces. In this way the Gad Naot module becomes a $\mathbb{Z}[T]$ module where $T$ is the two handles operator. Gad Naot discusses the situation if $\frac{1}{2}$ is added to the ground ring. Then it is easy to see that the resulting module is essentially the $h=0$ reduction of our skein module for the universal rank 2 Frobenius system. We call the resulting Frobenius algebra $\mathcal{F}_{G N}$. Note that labeling a component by $X$ corresponds 
to multiplication by $\frac{1}{2}$ and adding a trivial 1-handle to this component. Multiplication by $T$ in Gad Naot's module thus corresponds to multiplication by $4 t$ in our module.

The skein modules from [B], [N1] and [N2] actually are originally based on the 4-tube relation instead of the neck cutting relation. It has been proved in $[\mathrm{B}]$ that the neck cutting relation implies the 4 -tube relation. If 2 is invertible in $R$, as in $\mathbb{Z}\left[\frac{1}{2}, t\right]$, then the 4 -tube relation also imples the neck cutting relation. The 4 -tube relation is motivated from the Khovanov construction, giving the essential invariance of link homology under Reidemeister moves. But from the viewpoint of topological quantum field theory or Frobenius algebras the neck cutting relations seem more fundamental.

Following 2.2 we calculate for the Gad Naot modules $\varepsilon_{n}=0$ for $n$ even nonnegative integers and $\varepsilon_{n}=t^{\frac{n-1}{2}}$ for $n$ odd integers. Also $\mu(\Delta(1))=2 X$. Thus for a genus $g$ trivially colored surface by 5.3 , the image in $C\left(D^{3}\right)$ is

$$
\left(1+(-1)^{g+1}\right)^{g} t^{\frac{g-1}{2}} \in \mathbb{Z}[t],
$$

see $[\mathrm{N} 1]$.

Note that examples (a)-(c) define epimorphisms of Frobenius algebras

$$
\mathcal{F}_{\mathbf{U}} \rightarrow \mathcal{F}_{G N} \rightarrow \mathcal{F}_{B N}
$$

(d) Let $\mathcal{F}_{\{e\}}$ be the Frobenius algebra defined in 2.2 (b) for $G=\{e\}$ the trivial group. Then $A=R$ with identity $1=e$ and the neck cutting relation following $\Delta(1)=e \otimes e$ allows to replace a surface with the surface resulting from cutting the neck. Moreover, the trivially colored 2 -sphere is 1 in this case. Thus it follows that

$$
C\left(M, \alpha ; \mathcal{F}_{\{e\}}\right) \cong \mathbb{Z} H_{2}\left(M ; \mathbb{Z}_{2}\right)
$$

for each $\alpha$ with $j_{*}[\alpha]=0$. If $j_{*}[\alpha] \neq 0$ then $C\left(M, \alpha ; \mathcal{F}_{\{e\}}\right)=0$. It follows from 7.1 and 7.2 that $C\left(M, \alpha ; \mathcal{F}_{G}\right) \rightarrow \mathbb{Z} H_{2}\left(M ; \mathbb{Z}_{2}\right)$ is an epimorphism. Thus skein modules for finite group Frobenius algebras appear as natural deformations of the group algebra $\mathbb{Z} H_{2}\left(M ; \mathbb{Z}_{2}\right)$

9. Presentations of skein modules from incompressible surfaces. First we generalize an important observation of Asaeda and Frohman [AF]. Recall that a compression disk $D$ for a surface $S \subset M$ is a disk in $M$ such $D \cap S=\gamma$ where $\gamma$ is an essential curve on $S$. Then $\gamma$ is called a compression curve. The surface $S$ is called incompressible if there are no compression disks and no components which are 2-spheres bounding 3-balls in $M$. A surface which does not have any compression curves but possibly has 2 -sphere components bounding 3-balls in $M$ is called weakly incompressible. Colored surfaces are called (weakly) incompressible if their underlying topological surfaces are (weakly) incompressible. For each $\mathfrak{a} \subset A$ let $\mathcal{I}(M, \alpha, \mathfrak{a})$ denote the set of incompressible surfaces in $M$ bounding $\alpha$ with components colored by elements of $\mathfrak{a}$.

TheOrem 9.1. Let $A$ be generated by $\mathfrak{g} \subset A$. Then the skein module $C(M, \alpha)$ is generated by the images in $C(M, \alpha)$ of the set $\mathcal{I}(M, \alpha, \mathfrak{g}) \subset \mathcal{S}(M, \alpha, \mathfrak{g})$, which is called the set of marked incompressible surfaces.

Proof. By 8.1 the module is certainly generated by $\mathcal{S}(M, \alpha, \mathfrak{g})$, the set of marked surfaces. If such a surface $S$ is not weakly incompressible then there is a simple closed loop $\gamma$ on $S$ which bounds a disk $D$ in $M$ such that $D \cap F=\gamma$. Moreover, if $\gamma$ is not 2-sided on $S$ then 
the normal bundle of $\gamma$ in $M$ is nontrivial. But this is not possible since $\gamma$ bounds a disk in $M$. (The fact that orientability of $M$ is not relevant in this situation and the argument above have been pointed out to me by Charlie Frohman.) Thus we can apply a neck cutting relation and expand in terms of marked surfaces. After finitely many steps the surface will be weakly incompressible. Finally we can apply sphere relations to eliminate all 2-spheres which bound 3-balls in $M$.

Recall that a 3-manifold is irreducible if each 2-sphere in $M$ bounds a 3 -ball. It is a simple but important observation that for irreducible $M$ we can assume that neck cutting relations only apply to curves $\gamma$ which are essential on a colored surface $S$. In fact a neck cutting relation for an inessential loop on a surface is in patch notation:

$$
(a)=\sum_{i}\left(a u_{i}\right)\left(v_{i}\right)
$$

with the second patch indicating a 2-sphere, which bounds a 3-ball in $M$ because of irreducibility. But also

$$
\sum_{i}\left(a u_{i}\right)\left(v_{i}\right)=\sum_{i} \varepsilon\left(v_{i}\right)\left(a u_{i}\right)=\left(\sum_{i} \varepsilon\left(v_{i}\right) a u_{i}\right)=((I d \otimes \varepsilon) \circ \Delta(a))=(a)
$$

using the $R$-multi-linearity relations, sphere relations and equation (1).

Let $\mathfrak{p}=(\mathfrak{g}, \mathfrak{r})$ be as in section 8 . Let Let $\mathfrak{C}(M, \alpha, \mathfrak{p}) \subset \mathfrak{R}(M, \alpha, \mathfrak{p}) \cap R \mathcal{I}(M, \alpha, \mathfrak{g})$ be the submodule of $R \mathcal{I}(M, \alpha, \mathfrak{g})$, which is generated by all relations $\left(1^{\prime}\right)$ with all colored surfaces in $\mathcal{I}(M, \alpha, \mathfrak{g})$.

Next we define the tubing submodule

$$
\mathfrak{T}(M, \alpha, \mathfrak{g}) \subset R \mathcal{S}(M, \alpha, \mathfrak{g})
$$

of tubing relations in $M$. The importance of this module comes from the following:

MAIn TheOREM 9.2. Let $\mathcal{F}$ be a Frobenius algebra with presentation $\mathfrak{p}=(\mathfrak{g}, \mathfrak{r})$. Let $M$ be an irreducible 3-manifold with 1-manifold $\alpha \subset \partial M$. Then we have the isomorphism

$$
C(M, \alpha) \cong R \mathcal{I}(M, \alpha, \mathfrak{g}) /(\mathfrak{T}(M, \alpha, \mathfrak{g})+\mathfrak{C}(M, \alpha, \mathfrak{p}))=: \mathcal{D}(M, \alpha) .
$$

Note that in its definition $\mathcal{D}(M, \alpha)$ depends on the presentation $\mathfrak{p}$. We omit the presentation from the notation here because our result shows that the quotient actually does not depend on it.

EXAMPLE 9.3. If $\mathcal{F}$ is free with basis $\mathfrak{g}$ then for the usual presentation, $\mathfrak{C}(M, \alpha, \mathfrak{p})=0$. Then in 9.2 all relations necessary are the tubing relations involving incompressible surfaces marked by basis elements.

We now define the isomorphism of the theorem and the tubing submodule. First consider $S \in \mathcal{S}(M, \alpha, \mathfrak{g})$ and use 9.1 to expand a representative surface, also denoted $S$, as $R$-linear combination of incompressible marked surfaces. We do this in two steps.

Definition 9.4. Let $M$ be a 3-manifold with $\alpha \subset \partial M$ a closed 1-manifold. Let $\mathfrak{a} \subset A$. Then an a-pattern $\Gamma$ is a finite graph with (i) a decomposition of its vertex set into a disjoint union of two sets, the black and the white vertices, (ii) a coloring of the components of the graph by elements of $\mathfrak{a}$ and, (iii) a one-to-one correspondence between 
the black vertices and the components of an irreducible surface in $M$. The set of a-patterns is denoted $\mathcal{P}(M, \alpha, \mathfrak{a})$.

For $\mathfrak{g}$ a generating set we call a $\mathfrak{g}$-pattern just a pattern. Define the projection

$$
\mathfrak{h}: \mathcal{P}(M, \alpha, \mathfrak{a}) \rightarrow \mathcal{I}(M, \alpha, 1)
$$

by assigning to each pattern the incompressible surface defined by its black vertices with trivial coloring of its components.

Now each presentation $\mathfrak{p}=(\mathfrak{g}, \mathfrak{a})$ of the algebra $A$ of our Frobenius algebra defines a state sum map

$$
\mathcal{K}: \mathcal{P}(M, \alpha, \mathfrak{g}) \rightarrow R \mathcal{I}(M, \alpha, \mathfrak{g}) / \mathfrak{C}(M, \alpha, \mathfrak{p})
$$

Fix a Frobenius system for $\mathcal{F}$ such that

$$
\Delta(1)=\sum_{i=1}^{r} u_{i} \otimes v_{i} .
$$

Also choose orientations of the edges of $\Gamma$. Let a state $\sigma$ on $\Gamma$ assign to each edge of $\Gamma$ an element of $\{1, \ldots, r\}$. We calculate the state evaluation $\mathfrak{e}(\sigma) \in \mathcal{I}(M, \alpha, \mathfrak{g})$ as follows. If an edge $e$ is running from a vertex $v$ to a vertex $w$ and $\sigma(e)=i$ then assign to $v$ the element $u_{i}$ and to $w$ the element $v_{i}$. Finally assign the color $a$ of a component to any of its vertices. Multiply all the elements assigned to the vertices. Consider the incompressible surface determined by the black vertices. Use the relations of $A$ to expand the colors in terms of the generating set. This is only defined up adding elements of $\mathfrak{C}(M, \alpha, \mathfrak{p})$. The result is an element in $R \mathcal{I}(M, \alpha, \mathfrak{g}) / \mathfrak{C}(M, \alpha, \mathfrak{p})$ where the underlying surface is always the same but the colors in $\mathfrak{g}$ can change. Finally each white vertex $v$ is colored by some element $b_{v} \in A$. Multiply the element of $R \mathcal{I}(M, \alpha, \mathfrak{g}) / \mathfrak{C}(M, \alpha, \mathfrak{p})$ determined above by

$$
\prod_{v \text { white vertex }} \varepsilon\left(b_{v}\right) \in R
$$

The resulting element is $\mathfrak{e}(\sigma)$. The sum over all states $\sigma$ defines $\mathcal{K}(\Gamma)$. While $\mathfrak{e}(\sigma)$ in general will depend on choice of edge orientations the full state sum does not because of cocommutativity of $\Delta$.

Example 9.5. Consider the Bar-Natan system $\mathcal{F}_{B N}$ with Frobenius system defined by $\{(1, X),(X, 1)\}$. Then there are only two states that assign the dots to the different endpoints of edges. In this case the state itself can be identified with an orientation of the edges of the graph.

Next we assign to $S \in \mathcal{S}(M, \alpha, \mathfrak{g})$ elements of $\Gamma \in \mathcal{P}(M, \alpha, \mathfrak{g})$ such that $\mathcal{K}(\Gamma)$ are expansions of $S$. Note that given $S$ we can apply neck cut compressions until the resulting surface is weakly incompressible. Because the neck cuttings always increase the Euler characteristic by 2 this process stops after finitely many steps with a weakly incompressible surface with the history of the neck cuttings defining the edges of the corresponding graph.

Next consider the obvious map:

$$
\mathfrak{d}: \mathcal{P}(M, \alpha, \mathfrak{g}) \times \mathcal{P}(M, \alpha, \mathfrak{g}) \rightarrow R \mathcal{I}(M, \alpha, \mathfrak{g}) / \mathfrak{C}(M, \alpha, \mathfrak{p})
$$


defined by

$$
\left(\Gamma_{1}, \Gamma_{2}\right) \mapsto \mathcal{K}\left(\Gamma_{1}\right)-\mathcal{K}\left(\Gamma_{2}\right) .
$$

For each $S \in \mathcal{S}(M, \alpha, 1)$ consider all possible $\Gamma \in \mathcal{P}(M, \alpha, \mathfrak{g})$ assigned to $S$ using the construction above. Let

$$
\mathcal{D}(S) \subset \mathcal{P}(M, \alpha, \mathfrak{g}) \times \mathcal{P}(M, \alpha, \mathfrak{g})
$$

be the set defined by all possible pairs of elements constructed from $S$ in this way and by assigning arbitrary colors to the different components of $\Gamma_{1}$ and $\Gamma_{2}$ in an arbitrary way such that corresponding components are colored in the same way.

Definition 9.6. Let $\mathfrak{T}(M, \alpha, \mathfrak{g})$ be the submodule of $\mathcal{I}(M, \alpha, \mathfrak{g})$ which is generated by the union of all sets $\mathcal{K}(\mathfrak{d}(\mathcal{D}(S))$ for all $S \in \mathcal{S}(M, \alpha, 1)$.

Then by construction the map

$$
\mathfrak{t}: \mathcal{S}(M, \alpha, \mathfrak{g}) \rightarrow \mathcal{D}(M, \alpha)
$$

which chooses an expansion graph for $S$ and assigns to it the equivalence class of the state sum in $\mathcal{D}(M, \alpha)$ as described above, is well-defined.

Proof of Theorem 9.2. Using 8.1 we identify $C(M, \alpha)$ and $C(M, \alpha, \mathfrak{p})$. It is not hard to check that the relations $\left(1^{\prime}\right)-\left(3^{\prime}\right)$ from section 8 are in the kernel of the $R$-module map $\mathfrak{t}$ : $R \mathcal{S}(M, \alpha, \mathfrak{g}) \rightarrow \mathcal{D}(M, \alpha)$. For example consider a neck cutting relation. Then because the $\mathfrak{t}$-expansions are (by construction) independent of the way in which neck cutting relations are applied we can choose the expansions compatibly. It is here important that we only have to consider neck cuttings on essential curves. Also sphere relations will contribute a factor that also is picked up in the state sum. The relations $\left(1^{\prime}\right)$ induced from $\mathfrak{r}$ map to $\mathfrak{C}(M, \alpha, \mathfrak{p})$ under the $\mathfrak{t}$-expansions. Conversely, the inclusion $\mathcal{I}(M, \alpha, \mathfrak{g}) \rightarrow \mathcal{S}(M, \alpha, \mathfrak{g})$ defines the $R$-module map

$$
\mathfrak{s}: R \mathcal{I}(M, \alpha, \mathfrak{g}) \rightarrow C(M, \alpha, \mathfrak{p}) .
$$

It is immediate from the definitions that

$$
\mathfrak{T}(M, \alpha, \mathfrak{g})+\mathfrak{C}(M, \alpha, \mathfrak{p})
$$

is contained in the kernel and thus $\mathfrak{s}$ induces an $R$-module map

$$
\mathcal{D}(M, \alpha) \rightarrow C(M, \alpha, \mathfrak{p}),
$$

which is inverse to $t$.

REMARK 9.7. (a) Irreducibility is important in the discussion of skein modules of surfaces in 3-manifolds. This has first been pointed out in $[\mathrm{AF}]$. The result above is not true in general. For example one can show that in the Bar-Natan module of $M=\left(S^{1} \times S^{2}\right) \sharp\left(S^{1} \times\right.$ $\left.S^{2}\right)$ the connected sum sphere represents an element $S \in C(M)$ with $4 S=0$.

(b) For each $S_{1}, S_{2} \in \mathcal{I}(M, \alpha, 1)$ such that $\left[S_{1}\right]=\left[S_{2}\right] \in H_{2}\left(M, \alpha ; \mathbb{Z}_{2}\right)$ there exists $S \in \mathcal{S}(M, \alpha, 1)$ such that there exist $\left(\Gamma_{1}, \Gamma_{2}\right) \in \mathcal{D}(S)$ which satisfy $\mathfrak{h}\left(\Gamma_{i}\right)=S_{i}$ for $i=1,2$. This follows from 7.4. In particular $\mathcal{D}(S) \neq \emptyset$. Thus two incompressible surfaces within 
the same homology class give rise to relations

$$
\sum_{i=1}^{n_{1}} r_{i}^{1}\left(S_{1}, w_{i}^{1}\right)=\sum_{j=1}^{n_{2}} r_{j}^{2}\left(S_{2}, w_{j}^{2}\right)
$$

for $w_{i}^{1}: S_{1} \rightarrow \mathfrak{g}$ and $w_{j}^{2}: S_{2} \rightarrow \mathfrak{g}$ suitable $\mathfrak{g}$-colorings and $r_{i}^{1}, r_{2}^{j} \in R$ for $i=1, \ldots, n_{1}$ and $j=1, \ldots, n_{2}$.

ExAmple 9.8. Consider our version of the Gad Naot skein module as discussed in 8.3 (c) with $R=\mathbb{Z}$. Let $M=S^{3} \backslash \operatorname{int}(N(K))$ where $N(K) \subset S^{3}$ is a tubular neighborhood of a nontrivial knot $K \subset S^{3}$. and int is its interior. Then the boundary torus $S:=\partial N(K) \subset$ $M$ is an incompressible surface representing an element $T \in C(M)$. Let $g$ be the tunnel number of the knot $k$. Then attaching $g$ tubes to $N(K)$ bounds a handlebody in $M$. Thus by $g$ applications of the neck cutting relation, 8.3 (c) and $X^{2}=t$, we conclude

$$
\left(S, 2^{g} X^{g}\right)=\left(1+(-1)^{g}\right)^{g+1} t^{\frac{g}{2}} .
$$

Thus for $g$ even we get

$$
2^{g} t^{\frac{g}{2}} S=2^{g+1} t^{\frac{g}{2}}
$$

or

$$
2^{g} t^{\frac{g}{2}}(S-2)=0
$$

and for $g$ odd we get

$$
2^{g} t^{\frac{g-1}{2}}(S, X)=0 .
$$

The geometry of tunnel numbers of knots is highly nontrivial and one of the most interesting subjects of the classical topology of knots in 3-manifolds, see e.g. [Mo].

Acknowledgements. I would like to thank Masahico Saito for suggesting the study of skein modules of surfaces for general Frobenius algebras. I also would like to thank Carmen Caprau and Charlie Frohman for several interesting and helpful remarks. It has been Charlie's enthusiam that convinced me to proceed with this subject.

This research was supported by NSF grant DMS-0204627.

\section{References}

[A] L. Abrams, Two-dimensional topological quantum field theories and Frobenius algebras, J. Knot Theory and its Ramifications 5 (1996), 569-587.

[AF] M. Asaeda and C. Frohman, A note on the Bar-Natan skein module, math.GT/ $0602262 \mathrm{v} 1$.

[B] D. Bar-Natan, Khovanov homology for tangles and cobordisms, Geom. Topology 9 (2005), 1143-1199.

[C] C. Caprau, An sl(2) tangle homology and seamed cobordisms, math.GT/0707.3051v2.

[FK] C. Frohman and U. Kaiser, Homology of links in 3-manifolds, preprint, 2008.

$[\mathrm{H}] \quad$ N. Hitchin (Notes by David Calderbank) Frobenius manifolds, in: Gauge Theory and Symplectic Geometry, J. Hurtubise et al. (eds.), Kluwer 1995, 69-112.

[K] L. Kadison, New Examples of Frobenius Extensions, University Lecture Series 14, AMS, 1999. 
[Ka] U. Kaiser, Link Theory in Manifolds, Lecture Notes in Mathematics 1669, Springer Verlag, 1997.

[Kh] M. Khovanov, Link homology and Frobenius extensions, Fund. Math. 190 (2006), 179-190.

[Ko] J. Kock, Frobenius Algebras and 2D Topological Quantum Field Theories, Cambridge University Press, 2004.

[KL] C. Kearton and W. B. Lickorish, Piecewise linear critical levels and collapsing, Trans. AMS 170 (1972), 415-424.

[L] T. Le, The colored Jones polynomial and the A-polynomial of knots, Adv. Math. 207 (2006), 782-804.

[M] V. Manturov, The Khovanov complex for virtual links, J. Math. Sci. (N.Y.) 144 (2007), 4451-4467.

[Mo] M. Morimoto, On unknotting tunnels for knots, Math. Annalen 289 (1991), 143-167.

[N1] G. Naot, The universal Khovanov link homology theory, Alg. Geom. Top. 6 (2006), 18631892.

[N2] G. Naot, The universal sl(2) link homology, math.GT/0706.3680v1.

[P] J. Przytycki, Skein modules of 3-manifolds, Bull. Pol. Acad. Sci. 39 (1991), 91-100.

[T1] V. Turaev, The Yang Baxter equations and invariants of links, Inventiones Math. 93 (1988), 527-553.

[T2] V. Turaev, Quantum Invariants of Knots and 3-Manifolds, de Gruyter Studies in Mathematics 18, Walter de Gruyter, Berlin, 1994.

[TT] V. Turaev and P. Turner, Unoriented topological quantum field theory and link homology, Alg. Geom. Top. 6 (2006), 1069-1093. 
